\title{
Probing the Positions of TeO Moieties in the Channels of the MoVNbTeO M1 Catalyst: A Density Functional Theory Model Study
}

\author{
Juan Manuel Arce-Ramos ${ }^{1}$ (D) Graham Rugg $^{1}$ (D) $\cdot$ Alexander Genest $^{1,2}$ (D) Notker Rösch $^{2,3}$ (D)
}

Received: 17 November 2020 / Accepted: 11 January 2021 / Published online: 30 January 2021

(c) The Author(s) 2021

\section{Abstract}

With hybrid DFT calculations applied to periodic models of the bulk MoVNbTeO M1 catalyst, we examined how [TeO] ${ }^{2+}$ species in the hexagonal channels of this material stabilize nearby reduced metal centers. In particular, an S2(Mo) site, with adjacent $[\mathrm{TeO}]^{2+}$ moieties at both sides, is calculated to be reduced to $\mathrm{Mo}^{5+}$. The modeling study presented offers insight into how the redox behavior of $\mathrm{V}$ and Mo centers, a crucial aspect of the M1 catalyst for the selective partial oxidation of small hydrocarbons, may be fine-tuned via $\mathrm{TeO}$ moieties at various distances from the metal centers.

\section{Graphic Abstract}

$\mathrm{TeO}$ moieties in hexagonal channels, adjacent on either side of an S2(Mo) center, stabilize a gap state at the Mo center, facilitating its reduction to $\mathrm{Mo}^{5+}$.

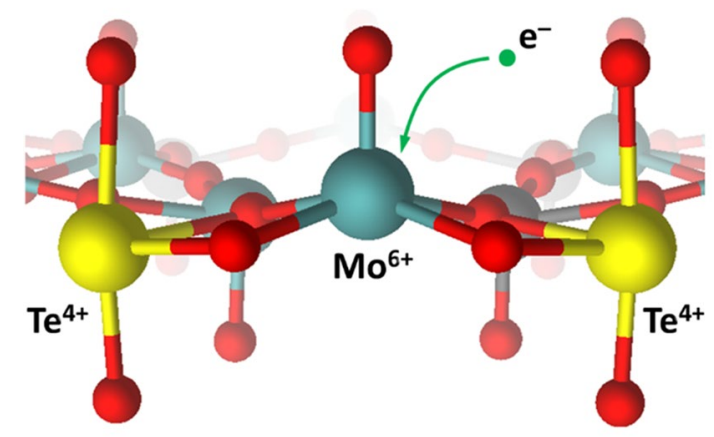

Keywords M1 catalyst $\cdot$ MoVNbTeO material $\cdot \mathrm{TeO}$ moieties $\cdot$ Location of TeO $\cdot$ Reduced metal centers $\cdot$ Electrostatic interaction · Hybrid DFT calculations

Alexander Genest

alexander.genest@tuwien.ac.at

Notker Rösch

roesch@mytum.de

1 Institute of High Performance Computing, Agency for Science, Technology and Research, 1 Fusionopolis Way, \#16-16 Connexis, Singapore 138632, Singapore

2 Institute of Materials Chemistry, Technische Universität Wien, Getreidemarkt 9, 1060 Vienna, Austria

3 Department Chemie and Catalysis Research Center, Technische Universität München, 85747 Garching, Germany

\section{Introduction}

The catalytic production of short-chain olefins is typically carried out by energy-intensive steam cracking [1]. Moreover, with the advent of pure-ethane crackers, olefins, like propylene, formerly available as side products, become relatively less easily accessible, stimulating the development of alternatives routes for producing these olefins [2]. The MoVNbTeO catalyst stands out as an alternative for producing short olefins through oxidative dehydrogenation $(\mathrm{ODH})$ of alkanes. This catalyst material is also promising 
in the production of acrylonitrile from propane [3, 4], avoiding propylene as used in the established process [5]. Two crystalline phases of the MoVNbTeO material have been reported to be important for the catalysis. The M1 phase is in charge of the activation of the alkane and the $\mathrm{ODH}$, while the $\mathrm{M} 2$ phase has been assigned an auxiliary function [6-8]. Therefore, the M1 phase is the only one required if one simply aims at an $\mathrm{ODH}$ process.

Since the discovery of the MoVNbTeO mixed-metal oxide catalyst [3], significant effort was devoted in understanding this catalyst. Studies in this field range from synthesizing MoVNbTeO and its variants [9-11], characterizing the structure of these materials [12-20], elaborating the catalytic mechanism [21-23], to elucidating how the metal centers and $\mathrm{V}^{5+} / \mathrm{V}^{4+}$ pairs are distributed in the material [24-28]. It is well accepted that the $\mathrm{V}^{5+}$ species are responsible for activating the alkanes over this catalyst [21,27, $29,30]$, yet clarifying their location remains as one of the challenges. For an efficient catalyst design it is important to understand the factors that determine the presence and distribution of $\mathrm{V}^{5+}$ species. A higher concentration of $\mathrm{V}^{5+}$ has been determined in the vicinity of hexagonal pores with decreased $\mathrm{TeO}$ content in the MoVNbTeO M1 catalyst [28]. $\mathrm{TeO}$ moieties have also been discussed to take an active role during the catalysis $[11,17,31]$.

In this letter, using a computational approach and model structures, we explore the local positions of Te centers in the hexagonal channels, and we examine how such centers affect the local lattice structure around neighboring metal centers and the electronic states of these sites.

In particular, we will address the effects of $\mathrm{TeO}$ moieties on sites $\mathbf{2}$ at the centers of the "pentameric units", Fig. 1, that are nearby according to experimental evidence $[14,16]$. According to our model calculations, reducing electrons in metal $d$-states are stabilized in the proximity of $[\mathrm{TeO}]^{2+}$ moieties. Overall, we are aiming at improving our understanding of the factors that affect the distributions of $\mathrm{V}^{4+}$ / $\mathrm{V}^{5+}$ centers, thus hoping for enabling the synthesis of more active/selective catalyst materials for oxidation processes.

Given the complexity of the material at hand, we focus on understanding the effect of $\mathrm{TeO}$ positions in the hexagonal channels of a bulk model. This admittedly idealized situation allows us to deduce the basic effects of intercalated $\mathrm{TeO}$. More complex situations, like the effect of a surface of MoVNbTeO, will be deferred to subsequent studies.

\section{Models and Methods}

We studied bulk models of the MoVNbTeO M1 oxide, for computational ease maintaining ideal three-dimensional periodicity. Here, we first present the considerations a
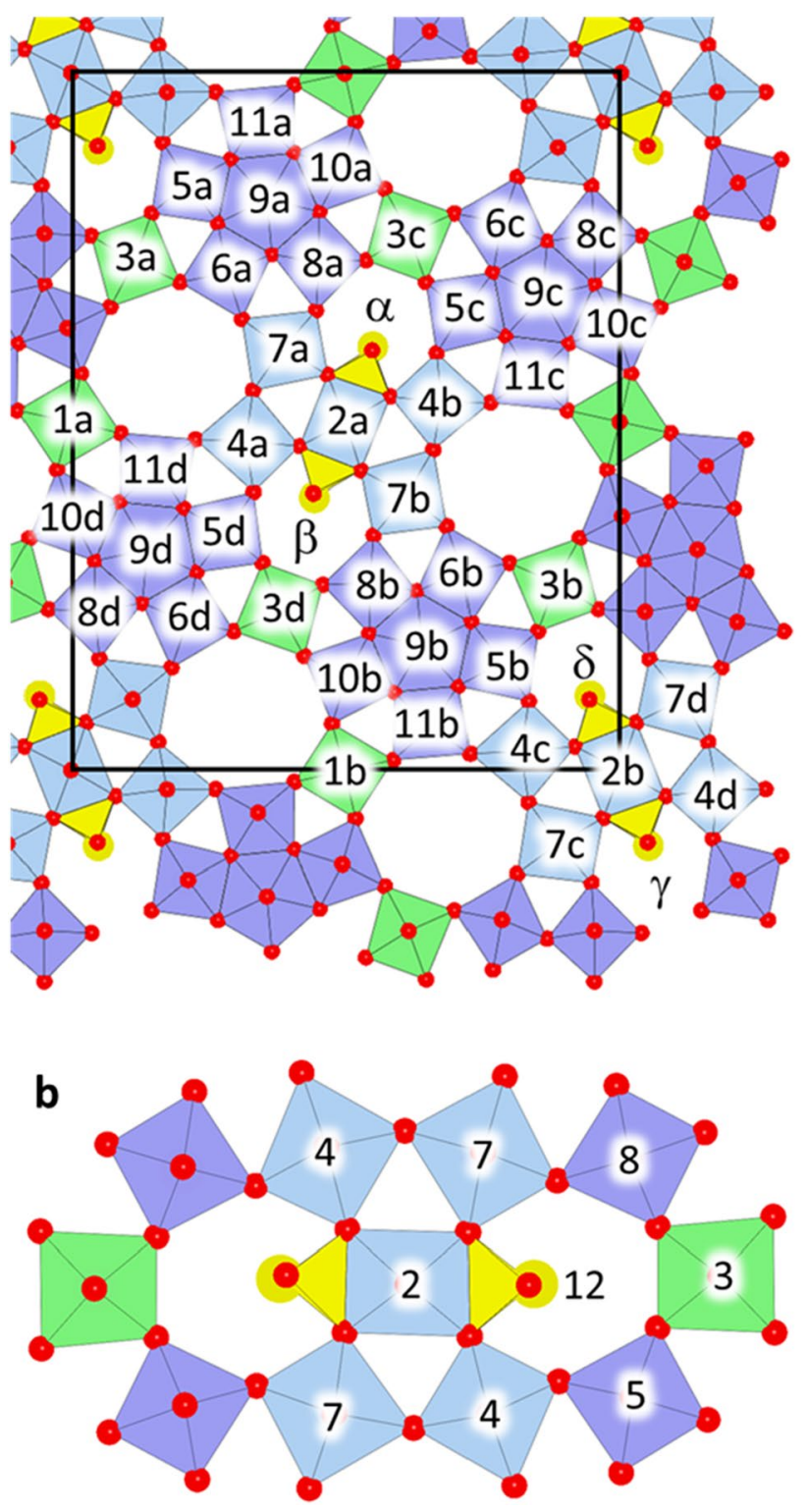

Fig. 1 a Sketch of an ideal MoVNbTeO M1 bulk structure, viewed in the [001] direction. The metal sites are marked by labels $\boldsymbol{n}$ (instead of common nomenclature Sn, Refs. [16, 18, 63]) with lower-case characters a- $d$ added to discriminate symmetry equivalent sites. The centers are rendered in yellow, exclusively occupying sites $\mathbf{1 2}$ in the hexagonal channels (HCs) $\alpha-\delta$. The positions $\mathbf{1 3}$ (not marked) in the heptagonal channels are empty in our model. Pentameric units, discussed as the catalytically active sites, are shown in blue, pentagonal units in purple, and so-called linker sites in green. Oxygen centers are indicated as red discs. b Close-up of the pentameric unit, bracketed by Te sites in the adjacent HCs. In our model the occupancy is 2(Mo), 4(Mo), and 7(V)

undertaken to build the bulk models and the nomenclature used throughout this work. We then describe the computational strategy; additional details may be found in the Electronic Supplementary Material (ESM, Online Resource 1). 
The average stoichiometry of the MoVNbTeO M1 material as reported by Li et al. is $\mathrm{Mo}_{30} \mathrm{~V}_{6} \mathrm{Te}_{3} \mathrm{Nb}_{4} \mathrm{O}_{115}$ [16]. However, the MoVNbTeO M1 catalyst has been successfully synthesized with a higher $\mathrm{V}$ content $[18$, 26]. $\mathrm{TeO}$ moieties partially occupy both heptagonal and hexagonal channels, although higher occupancies of Te have been reported in the hexagonal channels $[14,16]$. We chose to model the M1 bulk by fixing the stoichiometry at $\mathrm{Mo}_{26} \mathrm{~V}_{10} \mathrm{Nb}_{4} \mathrm{Te}_{4} \mathrm{O}_{116}$, with a monolayer unit cell of that composition [28].

Following our previous work [28, 32], we label inequivalent sites of MoVNbTeO as $\mathbf{1}, \mathbf{2}, \mathbf{3}, \ldots, \mathbf{1 2}$, Fig. 1, shortened from the commonly accepted nomenclature S1, S2, S3, ..., S12 [16]. The pentameric units, formed by sites $2(\mathrm{~V}), \mathbf{4}(\mathrm{V})$, and $\mathbf{7}(\mathrm{Mo})$ in our models, have been discussed as active sites of the catalyst $[21,22]$. We specify sites of the same type by adding a lower case letter, e.g., $\mathbf{3 a}, \mathbf{3 b}$, $\mathbf{3 c}$, and $\mathbf{3 d}$ and we designate the various distributions of $\mathrm{V}^{4+}$ sites by a concatenated label that lists all reduced sites. For example, the label 1ab3abcd7abcd indicates that reducing electrons are located at all sites $\mathbf{1}, \mathbf{3}$, and 7, 10 electrons in total $[28,32]$. These latter sites have been reported with the highest $\mathrm{V}$ occupancies for the $\mathrm{MoVNbTeO}$ catalyst used in experiments $[14,18]$ and we thus selected them as being occupied by $\mathrm{V}$ centers in all our models. The choice of 10 reducing electrons per unit cell, at centers $\mathrm{V}^{4+}$, entails that initially small polarons are formed primarily at $\mathrm{V}$ sites [14], reflecting the higher ionization potential of $\mathrm{V}^{4+}$ compared to that of $\mathrm{Mo}^{5+}[33]$.

In agreement with experimental observations [14, 17], we initially placed the Te centers, in the form of $[\mathrm{TeO}]^{2+}$ moieties, at all positions $\mathbf{1 2}$ in the hexagonal channels, HCs, Fig. 1. Each HC is formed by the sites $\mathbf{2}, \mathbf{3}, \mathbf{4}, \mathbf{5}$, $\mathbf{7}$, and $\mathbf{8}$, Fig. 1b. In consequence, the channels of the bulk models are filled by stacks along the $c$ direction, i.e., "chains" of $[\mathrm{TeO}]^{2+}$ moieties oriented perpendicular to the $a b$ plane.

As we probed the stability of the $\mathrm{TeO}$ chains at various positions within the four HCs $\alpha-\delta$, Fig. 1 (per unit cell), we found it necessary to identify various structure motifs. We refer to a generic configuration as $[\boldsymbol{\alpha}, \boldsymbol{\beta}][\boldsymbol{\gamma}, \boldsymbol{\delta}]$, where the Greek characters are placeholders for the labels of the metal sites closest to the Te centers. The channel identifiers are grouped in pairs, bracketing one of the two pentameric units, Fig. 1b. We consider site $\boldsymbol{n}$ to be close to a Te center if two equatorial oxygen centers bonded to such a site form Te-O contacts shorter than $220 \mathrm{pm}$. For example, in the configuration $[\mathbf{2}, \mathbf{7}][\mathbf{3}, \mathbf{2}]$, the $\mathrm{TeO}$ moieties in the pair $\alpha$ and $\beta$ of HCs, bracketing the central pentameric unit, Fig. 1 , are close to the sites $\mathbf{2 a}$ and $\mathbf{7 b}$. The $\mathrm{TeO}$ moieties in the pair $\gamma$ and $\delta$ of $\mathrm{HCs}$, bracketing the other pentameric unit, are located close to sites $\mathbf{3 a}$ and $\mathbf{2} \mathbf{b}$, respectively.
Unless otherwise stated, all reported energies were obtained from electronic structure calculations using the software CRYSTAL14 [34], applying the hybrid DFT approach B3LYP [35], supplemented by the dispersion corrections D2 [36]. This electronic structure method offers a sufficient correction of self-interaction artifacts [37, 38], otherwise typical of regular GGA DFT functionals [39], thus affording a suitable localization of electrons at reduced $\mathrm{V}$ and Mo complexes [40]. Integrations over the Brillouin zone were carried out with a $1 \times 1 \times 4 k$-grid, following the scheme of Monkhorst-Pack [41]. Atomic charges were obtained from a Hirshfeld population analysis as implemented in the program CRYSTAL, with default parameters [42].

In the MoVNbTeO material, $\mathrm{V}$ (or Mo) centers are expected to exhibit electronic configurations $d^{0}$, when oxidized, or $d^{1}$, when reduced. For computational simplicity, we considered situations only where the spins of all reducing electrons are oriented parallel to each other (ferromagnetic spin orientation); low energy variations, $<8 \mathrm{~kJ} \mathrm{~mol}^{-1}$, resulted with antiferromagnetic spin orientations [32].

As in our previous studies on Mo- $\mathrm{V}$ mixed metal oxides [28, 32, 43], we used the all-electron split-valence basis sets 86-411d31G [44] and 8-411d1 [45] for $\mathrm{V}$ and $\mathrm{O}$ atoms, respectively. For Mo and $\mathrm{Nb}$ atoms, we chose the valence basis sets 311(d31)G [46] and 31(31d)G [47] in combination with Hay-Wadt small-core relativistic effective core potentials. For the Te atoms, we used a small-core relativistic effective core potential with the basis set m-pVDZ [48].

Projected densities of states were obtained with a $2 \times 2$ $\times 8 k$-point mesh and the corresponding density-weighted average position of gap states was calculated as shown previously [32]. Prior to the CRYSTAL/B3LYP structure optimizations, we generated approximate structures using the plane-wave code VASP [49-52] and the PBE + U electronic structure method [53,54], as described in the ESM (Online Resource 1) and elsewhere [28].

\section{Results and Discussion}

While the M1 phase of the MoVNbTeO bulk has on average symmetry Pba2, as determined by XRD Rietveld refinements [16]. Probing various $\mathrm{TeO}$ placements within the HCs may break the symmetry of the unit cell, entailing degenerate structures. Reducing the symmetry of the unit cell from $P b a 2$ to $P c$, for instance, leads to two degenerate structures related by one of the glide mirrors. In the present work, we only calculated those structures that are symmetry-inequivalent. 


\subsection{Locations of TeO Moieties and Their Influence on Neighboring Sites}

Next, we will assess the effects of the $\mathrm{TeO}$ location within the HCs. First, we explore how the structure and the stability of the system varies with the position of the $\mathrm{TeO}$ moieties within the HCs. Then we examine the influence of these $\mathrm{TeO}$ moieties on the reducibility of neighboring metal sites.

With the HCs, we determined stable locations for the $\mathrm{TeO}$ moieties near the sites $\mathbf{2}(\mathrm{Mo}), \mathbf{3}(\mathrm{V})$, and $\mathbf{7}(\mathrm{V})$, Fig. 2 and Table 1. These sites occur in the structure combinations $[\mathbf{2}, \mathbf{2}],[\mathbf{2}, 3],[\mathbf{2}, \mathbf{7}]$, and [7,7] in pairs of HCs bracketing a single pentameric unit, Fig. 2. Framework views of configurations included in Table 1 are shown in Fig. S1 (Online Resource 1). We probed also other locations for the $\mathrm{TeO}$ moieties, e.g., near 4(Mo), $\mathbf{5}(\mathrm{Mo})$, or $\mathbf{8}(\mathrm{Mo})$, but these locations were either substantially less stable or, during optimization, the $\mathrm{TeO}$ moieties returned to locations already known. $\mathrm{TeO}$ moieties have been reported to prefer sitting near V (rather than Mo) octahedra in the HCs of the M2 phase of MoVNbTeO [55]; it was suggested that the displacement of Te towards $\mathrm{V}$ is associated with the stronger repulsion between Te and Mo centers, due to their higher charges. Note that these charges are formal only. A Hirshfeld population analysis [42] of the structure [2,2][2,2] revealed that typical charges for $\mathrm{V}^{5+}, \mathrm{V}^{4+}, \mathrm{Mo}^{6+}, \mathrm{Mo}^{5+}$, and $\mathrm{Te}^{4+}$ are $2.32 e, 2.18 e, 2.37 e, 2.20 e$, and $2.33 e$, respectively. These charges are much lower than the various formal oxidation states. The formally highest oxidation state of the three elements $\mathrm{Mo}, \mathrm{V}$, and Te are rather similar in charge, and so are the reduced states, which are roughly $0.15 e$ lower in charge. This difference may be a driving force to reduce interactions when two metal centers are nearby, leading to a lower Coulomb repulsion due to the reduction of the oxidation state. This aspect should be kept in mind when we will be invoking Coulomb interactions in the following.

In agreement with a previous study [28], two $\mathrm{TeO}$ moieties bracketing site 2(Mo), [2,2], render this Mo center reduced, at the expense of the centers $\mathbf{1}(\mathrm{V})$, Table 1 . In all other placements of $\mathrm{TeO}$ moiety studied, the $\mathrm{V}^{4+}$ centers remain as originally selected (see above), i.e., the polaron distribution 1ab3abcd7abcd is maintained.

All energies in Table 1 are with respect to the symmetric configuration, $[\mathbf{2}, \mathbf{2}][\mathbf{2}, \mathbf{2}]$ with the alternate polaron distribution 2ab3abcd7abcd. We note, however, that this is not the most stable polaron distribution in which two $\mathrm{TeO}$ moieties bracket 2(Mo) centers, which was assessed elsewhere [28].

Inspecting Table 1 , we deduce the stability trend $[2,2]>[2,3],[3,2]>[2,7],[7,2]>[7,7]$ among the various

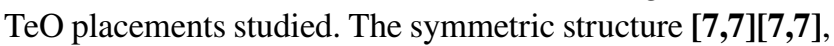
with all $\mathrm{TeO}$ moieties placed next to the reduced centers $7(\mathrm{~V})$, was determined as the least stable one, $66 \mathrm{~kJ} \mathrm{~mol}^{-1}$ higher in energy than the configuration $[2,2][2,2]$. The
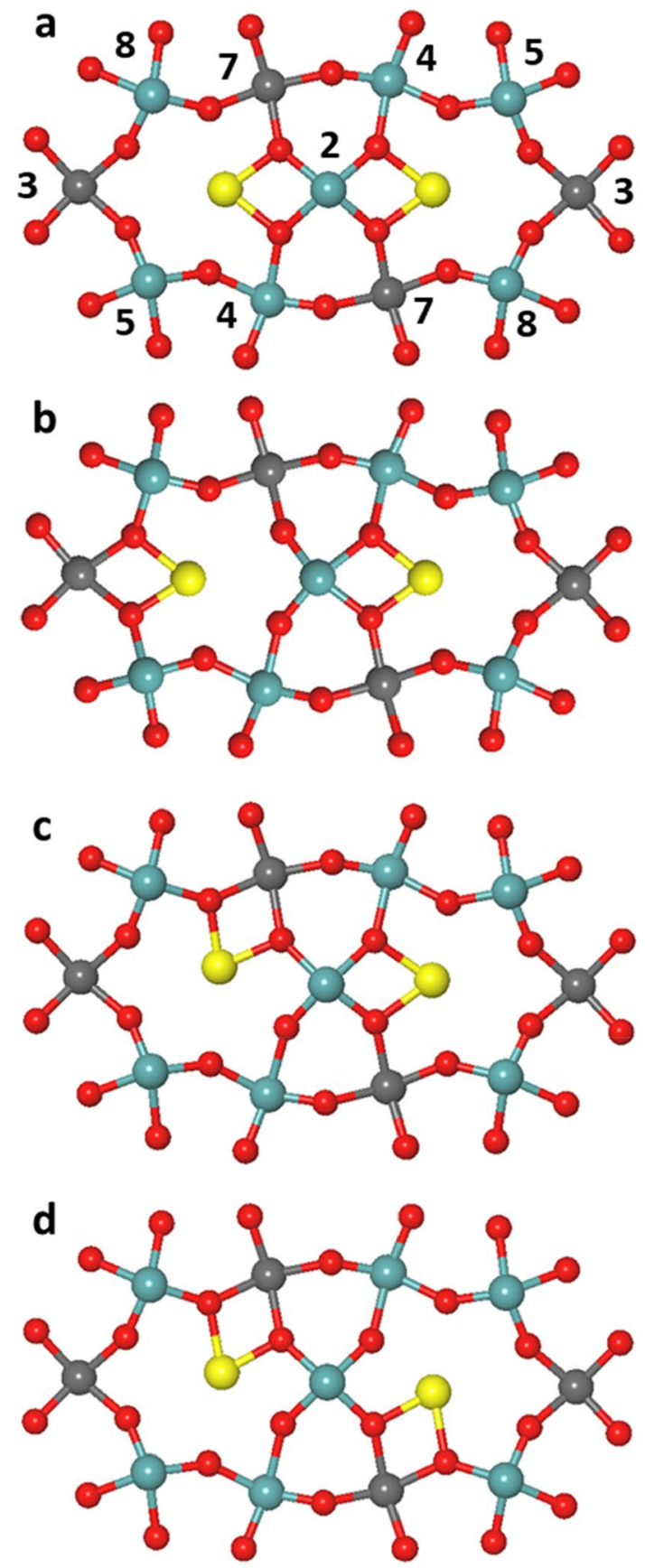

Fig. 2 Various placements for the TeO moieties within pairs of HCs bracketing a pentameric unit centered at site $\mathbf{2 b}$. For clarity, metal sites are labeled by $\boldsymbol{n}$, reduced from the common nomenclature Sn . Stable TeO location combinations are: a [2,2], b [3,2], c [7,2], and d $[7,7]$. Mo, $\mathrm{V}, \mathrm{Te}$ and $\mathrm{O}$ atoms are shown as teal, gray, yellow, and red spheres. Te-O bonds longer than $220 \mathrm{pm}$ are not shown

configurations $[7,2][7,2]$ and $[2,7][7,2], 8 \mathrm{~kJ} \mathrm{~mol}^{-1}$ more stable than [7,7][7,7], have one $\mathrm{TeO}$ moiety near a $7(\mathrm{~V})$ center while maintaining the $\mathrm{TeO}$ moiety in the neighboring $\mathrm{HC}$ at the center 2(Mo). Placing one or two of the $\mathrm{TeO}$ moieties across the pentameric unit next to a site $3(\mathrm{~V})$, producing 
Table 1 Relative energies $E, \mathrm{~kJ} \mathrm{~mol}^{-1}$, for various locations of the $\mathrm{TeO}$ moieties described by the labels $[\boldsymbol{\alpha}, \boldsymbol{\beta}][\boldsymbol{\gamma}, \boldsymbol{\delta}]$, where each Greek symbol represents a channel, Fig. 1

\begin{tabular}{llllll}
\hline Label & $d^{1 \text { a }}$ & & & \\
\cline { 2 - 5 } & $\mathbf{1}$ & $\mathbf{2}$ & $\mathbf{3}$ & $\mathbf{7}$ & $E$ \\
\hline$[\mathbf{7 , 7 ]}[\mathbf{7 , 7 ]}$ & $\mathrm{ab}$ & & abcd & abcd & 66 \\
{$[\mathbf{7 , 2}][\mathbf{7 , 2}]$} & $\mathrm{ab}$ & & abcd & abcd & 58 \\
{$[\mathbf{2 , 7 ] [ 7 , 2 ]}$} & $\mathrm{ab}$ & & abcd & abcd & 58 \\
{$[\mathbf{2 , 7 ]}[\mathbf{3 , 2}]$} & $\mathrm{ab}$ & & abcd & abcd & 53 \\
{$[\mathbf{2 , 3}][\mathbf{3 , 2}]$} & $\mathrm{ab}$ & & abcd & abcd & 40 \\
{$[\mathbf{2 , 2}][\mathbf{2 , 2}]^{b}$} & & ab & abcd & abcd & 0 \\
\hline
\end{tabular}

For a framework view of each structure in this table, see Fig. S1 (Online Resource 1)

${ }^{a}$ Metal centers with $d^{1}$ configuration, i.e., reduced or polaron centers, identified with the lower-case identifier $\mathrm{a}, \mathrm{b}, \mathrm{c}$, or $\mathrm{d}$; $\mathrm{V}$ at sites $\mathbf{1}, \mathbf{3}$, and 7; Mo at sites 2

${ }^{\mathrm{b}}$ Configuration used as reference

Table 2 Difference between trans-located equatorial 2(Mo)-O bonds, $\delta_{2}(\boldsymbol{n}-\mathbf{2}-\boldsymbol{n}$ '), for configurations displayed in Table 1

\begin{tabular}{lcccc}
\hline Structure & $\mathbf{7 a - 2 a - 7 b}$ & $\mathbf{4 a - 2 a - 4 b}$ & $\mathbf{7 c - 2} \mathbf{b}-\mathbf{7 d}$ & $\mathbf{4 c - 2 b - 4 d}$ \\
\hline$[\mathbf{7 , 7}][\mathbf{7 , 7 ]}$ & 0.0 & 0.0 & 0.0 & 0.1 \\
{$[\mathbf{7 , 2}][\mathbf{7 , 2}]$} & 6.3 & 22.9 & 6.3 & 22.9 \\
{$[\mathbf{2 , 7 ]}[\mathbf{7 , 2}]$} & 2.6 & 25.1 & 2.7 & 25.1 \\
{$[\mathbf{2 , 7 ]}[\mathbf{3 , 2}]$} & 2.2 & 29.2 & 25.3 & 24.7 \\
{$[\mathbf{2 , 3}][\mathbf{3 , 2}]$} & 26.2 & 27.8 & 26.2 & 27.8 \\
{$[\mathbf{2 , 2}][\mathbf{2 , 2}]$} & 0.0 & 0.0 & 0.0 & 0.0 \\
\hline
\end{tabular}

The centers $\boldsymbol{n}, \boldsymbol{n}^{\prime}$ and the corresponding $\mathbf{2}(\mathrm{Mo})$ center are indicated in each column. Values given in $\mathrm{pm}$

configurations $[2,7][3,2]$ and $[2,3][3,2]$, increases the stability of the unit cell by $13 \mathrm{~kJ} \mathrm{~mol}^{-1}$ and $26 \mathrm{~kJ} \mathrm{~mol}^{-1}$, respectively, relative to $[7,7][7,7]$.

To quantify how varying the $\mathrm{TeO}$ location affects the local structure of the $2(\mathrm{Mo})$ centers, we introduce the parameter $\delta_{2}\left(\boldsymbol{n}-\mathbf{2}-\boldsymbol{n}^{\prime}\right)$ which measures the (absolute) difference between trans located equatorial 2(Mo)-O bonds, Table 2. In a general manner, $\boldsymbol{n}$ and $\boldsymbol{n}$ ' refer to sites $\mathbf{4}$ or 7 , neighboring the oxygen atoms of these trans located bonds. When appropriate, we use both specific site labels, e.g. $\delta_{2}(\mathbf{7 a}-\mathbf{2 a}-7 \mathbf{b})$, indicating $\mathbf{7 a}(\mathrm{V})$ and $\mathbf{7 b}(\mathrm{V})$ as metal neighbors of the $\mathbf{2 a}(\mathrm{Mo})-\mathrm{O}$ bonds. For reduced $\mathbf{2}(\mathrm{Mo})$ centers, [2,2][2,2], the values $\delta_{2}(\mathbf{4}-\mathbf{2}-\mathbf{4})$ and $\delta_{2}(\mathbf{7}-\mathbf{2}-\mathbf{7})$ are all zero, indicating a symmetric octahedron with 2 (Mo) located at the intersection of the two diagonals, Table 2.

Such a symmetric environment of a metal center in an oxide normally is taken to indicate a $d^{1}$ metal center $[16,56]$. In contrast, if the central metal moiety is located away from the intersection of the two diagonals, then one invokes the pseudo Jahn-Teller concept and infers an oxidized 2(Mo) center as a consequence of the metal $d^{0}$ configuration [57]. The latter case is associated with larger values of $\delta_{2}$, in the range of 22-30 pm for one or both of the 4-2-4 or 7-2-7 diagonals, Table 2. In configuration $[7,7][7,7]$, the local structures, as indicated by $\delta_{2}=0 \mathrm{pm}$ in all four distinct $\boldsymbol{n - 2 - n}$ ' diagonals, are at variance with the pseudo Jahn-Teller concept [57], given that both metal centers $\mathbf{2 a}(\mathrm{Mo})$ and $\mathbf{2 b}(\mathrm{Mo})$ are oxidized. This computational result might be a consequence of the symmetrically located $\mathrm{TeO}$ moieties across the pentameric unit.

Configurations $[7,2][7,2]$ and $[2,7][7,2]$ feature oxidized 2(Mo) centers with an asymmetric environment, in agreement with the pseudo Jahn-Teller concept [57], with $\delta_{2}(\mathbf{4}-\mathbf{2}-\mathbf{4})=23-25 \mathrm{pm}$ and $\delta_{2}(\mathbf{7}-\mathbf{2}-\mathbf{7})=3-6 \mathrm{pm}$. While the $2(\mathrm{Mo})$ center is displaced towards one of the $4(\mathrm{Mo})$ on the diagonal 4-2-4, it remains close to the center of the diagonal 7-2-7. The direction of the distortion for $d^{0}$ metal centers may be due to an effective repulsion between metal ions, as previously postulated [58]. The distortion of 2(Mo) directed towards one of the vertexes of the diagonal 4-2-4 is likely due to the proximity of the centers Te and $\mathbf{2}(\mathrm{Mo})$. When the $\mathrm{TeO}$ moiety is located next to $\mathbf{2}(\mathrm{Mo})$, the center 2(Mo) shifts towards the opposite edge of the octahedron, Fig. 2b. For TeO located next to $7(\mathrm{~V})$, the 2 (Mo) moiety is displaced towards the site $\mathbf{4}(\mathrm{Mo})$ across the HC, Fig. 2c.

The centers 2(Mo) show strong off-center displacements in the configurations [2,3] and [3,2]. Sites $\mathbf{2 a}$ or $\mathbf{2 b}$ of $[\mathbf{2 , 3}$ $[3,2]$ and site $2 b$ of $[2,7][2,3]$ feature values of $\delta_{2}(\mathbf{4}-\mathbf{2}-\mathbf{4})$ and $\delta_{2}(\mathbf{7 - 2 - 7})$ of $25-29 \mathrm{pm}$, Table 2. These 2(Mo) centers are displaced towards the $\mathrm{HC}$ in which the $\mathrm{TeO}$ is located near 3(V), Fig. 2b. Especially those equatorial 2(Mo)-O bonds are elongated where the oxygen atoms are interacting with the $\mathrm{TeO}$ moiety while the other $2(\mathrm{Mo})-\mathrm{O}$ bonds are shortened, Figure S2 (Online Resource 1). Again, it appears as if an effective repulsion is at work between the Te and Mo metal ions, inducing a lattice distortion around these $\mathbf{2}(\mathrm{Mo})$ centers. Indeed, the deviations of $\mathbf{2}(\mathrm{Mo})$ from its central position were previously discussed as a cation-cation repulsion between site 2 and Te [59]. The asymmetric lattice distortion around site $\mathbf{2 a}(\mathrm{Mo})$ in the configuration $[\mathbf{2}, \mathbf{7}][\mathbf{2}, \mathbf{3}]$ along one diagonal, $\delta_{2}(\mathbf{4 a}-\mathbf{2 a}-\mathbf{4 b})=29 \mathrm{pm}$ and $\delta_{2}(\mathbf{7} \mathbf{a}-\mathbf{2 a}-7 \mathbf{b})=2 \mathrm{pm}$, may be rationalized in the same way as discussed above for the configurations $[\mathbf{7 , 2}][\mathbf{7 , 2}]$ and $[\mathbf{2 , 7 ]}$ $[7,2]$.

For the material $\mathrm{MoVO}_{\mathrm{x}}$, it was previously analyzed how the peak energies of the gap states (due to centers $\mathrm{V}^{4+}$ ) in the atom-projected density of states (pDOS) vary with the type of the reduced center, i.e., $\mathbf{1}(\mathrm{V}), \mathbf{3}(\mathrm{V})$, or $7(\mathrm{~V})$, as well as with the number of polaron-polaron interactions [32]. The latter aspect had been inferred as consequence of lattice distortions where a $\mathrm{MoO}_{6}$ moiety is bracketed between immediately adjacent centers $\mathrm{V}^{4+}$, 
due the larger ionic radius of these species in comparison with $\mathrm{V}^{5+}$. We analyzed the effect of these polaron-polaron interactions on the stability of MoVNbTeO elsewhere [28].

For the MoVNbTeO material we determined that the density-weighted average energy, $\varepsilon(\boldsymbol{n})$, of the gap states of reduced sites $\boldsymbol{n}$ is affected by the presence of the TeO moieties. In fact, $\varepsilon(\boldsymbol{n})$ shifts by $0.3-0.4 \mathrm{eV}$ to a lower energy if a $\mathrm{TeO}$ moiety is nearby, Table $\mathrm{S} 1$ (Online Resource 1). For instance, polarons with adjacent $\mathrm{TeO}$ moieties, $\mathbf{7 a}(\mathrm{V})$ and $\mathbf{7 c}(\mathrm{V})$ in configuration $[\mathbf{7 , 2}][\mathbf{7 , 2}]$ as well as $\mathbf{7 b}(\mathrm{V})$ and $\mathbf{7 c}(\mathrm{V})$ in configuration [2,7][7,2], Fig. 1, exhibit gap states that are about $0.3-0.4 \mathrm{eV}$ lower than the gap states of other polarons at 7(V) centers, Table S1 (Online Resource 1). Similar effects may be observed for the polaron $\mathbf{3 a}(\mathrm{V})$ in the configuration $[2,7][3,2]$ as well as the polarons $\mathbf{3 a}(\mathrm{V})$ and $\mathbf{3 d}(\mathrm{V})$ in the configuration $[\mathbf{2 , 3}][\mathbf{3 , 2}]$. Polaron-induced gaps states at lower energies in the pDOS may be interpreted to indicate a more facile reduction of those $\mathrm{V}$ centers that have a $[\mathrm{TeO}]^{2+}$ moiety nearby.

\subsection{Effect of the TeO Position on the Reducibility of Metal Centers}

Thus far, we discussed how a $\mathrm{TeO}$ moiety enhances the reducibility of $\mathrm{V}$ centers in their vicinity. Even more drastic is the concerted effect of two adjacent $\mathrm{TeO}$ moieties on a $\mathbf{2}(\mathrm{Mo})$ center in the configuration $[\mathbf{2}, \mathbf{2}][\mathbf{2}, \mathbf{2}]$ which results in a reduction of the site $\mathbf{2}(\mathrm{Mo})$ at the center of the pentameric unit. In fact, this configuration has the lowest energy among the configurations studied here, Table 1.

In the following, we explore whether and how shifting the $\mathrm{TeO}$ moieties within the hexagonal channels affects the oxidation state of the metal centers that form these channels. Such effects, including a relocation of the polarons there, could be due to the charge of $\mathrm{TeO}$ or a change in the bonding of equatorial oxygen centers of 2(Mo). Recall that, following the pseudo Jahn-Teller concept [57], reduced metal centers entail a more symmetric local environment in comparison to the analogous oxidized centers. We examined this question by carrying out two computer experiments where we shifted a $\mathrm{TeO}$ moiety in a step-wise fashion in the (001) within the HC, first admitting a local relaxation of centers other than $\mathrm{TeO}$ and subsequently fixing the geometry of all centers.

\subsubsection{Scanning TeO Positions on a 2-Dimensional Grid with Partial Structure Relaxation}

The hexagonal channel (HC) $\delta$ is formed by the sites 2b(Mo), 3b(V), 4c(Mo), 5b(Mo), 7d(V), and $8 d(M o)$, Fig. 3. For varying the positions of the $\mathrm{TeO}$ moiety inside the $\mathrm{HC} \delta$, we used a 2-dimensional grid in the (001) plane defined by the Te center in the configuration $[\mathbf{2 , 2}][\mathbf{2}, \mathbf{2}]$. The grid, with a step size of $20 \mathrm{pm}$, comprised only points in the $\mathrm{HC}$ where the distance between the Te atom and any of the equatorial oxygen atoms is at least $180 \mathrm{pm}$. On this grid, the $\mathrm{TeO}$ moiety was shifted as rigid structure from its initial position. In addition, we fixed the coordinates of the two $\mathrm{Nb}$ centers at sites $9 \mathbf{a}$ and $9 \mathbf{b}$, Fig. 1a, which reside farthest in the unit cell from the $\mathrm{HC}$ in question; this prevents a possible translation of the whole unit cell with respect to TeO. All other atoms were allowed to relax. We assessed the electronic configuration, $d^{0}$ or $d^{1}$, of the metal centers forming the $\mathrm{HC}$ by inspecting their spin densities $\rho$.

The reduced state, $d^{1}$, of the site $2 \mathbf{b}(\mathrm{Mo})$ was maintained for more than half of the $\mathrm{TeO}$ grid positions, cf. black dots in Fig. 3. When the TeO moiety is close to $\mathbf{5 b}(\mathrm{Mo})$ or $\mathbf{8 d}(\mathrm{Mo})$, at $\sim 370 \mathrm{pm}$ from $\mathbf{2 b}(\mathrm{Mo})$, the reducing electron is shifted to one of these latter sites, Fig. 3. The electronic configuration of sites $3 b(V), 4 c(M o)$, and $7 d(V)$ remained unchanged for all $\mathrm{TeO}$ positions probed. Apparently, electrostatic interactions trigger the reduction of metal sites in the wall of the $\mathrm{HC}$, at distances beyond direct bonds between a $\mathrm{TeO}$ moiety and an octahedron $\mathbf{2 b}(\mathrm{Mo})$. These conclusions agree with TEM results where (in the related M2 material) Te was preferentially found close to cations of smaller charge; this result was interpreted as a minimization of the cation-cation repulsion [55].

Relocating the reducing electron originating from $2 b(\mathrm{Mo})$ produces a "ridge" in the potential energy surface of at least $80 \mathrm{~kJ} \mathrm{~mol}^{-1}$ above the energy minimum, Fig. S3 (Online Resource 1). The local potential energy wells near $\mathbf{5 b}(\mathrm{Mo})$

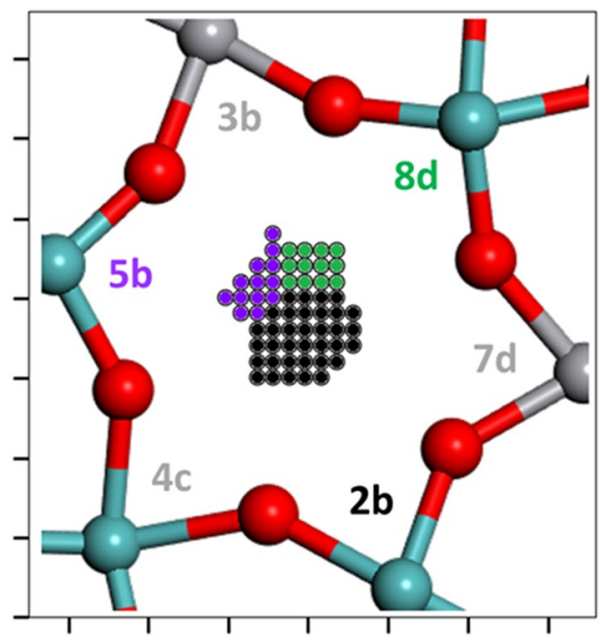

Fig. 3 Exploring the consequences of varying the position of the $\mathrm{TeO}$ moieties in the hexagonal channel. Depending on the color of the grid positions, black, purple, or green, the $d^{1}$ configuration is at the center, $\mathbf{2 b}(\mathrm{Mo}), \mathbf{5} \mathbf{b}(\mathrm{Mo})$, or $\mathbf{8 d}(\mathrm{Mo})$, respectively. For the total energy changes involved, see Fig. S3 (Online Resource 1). The initial structure used was $[\mathbf{2 , 2}][\mathbf{2 , 2}]$; see text for further details regarding the structures. The tick marks on the axes indicate increments of $100 \mathrm{pm}$ 
and $\mathbf{8 d}(\mathrm{Mo})$ are estimated to lie $\sim 35 \mathrm{~kJ} \mathrm{~mol}^{-1}$ relative to the initial configuration [2,2][2,2], Fig. S3 (Online Resource 1). At these latter $\mathrm{TeO}$ locations, the polaron initially at $\mathbf{2 b}(\mathrm{Mo})$, is relocated either to $\mathbf{5 b}(\mathrm{Mo})$ or $\mathbf{8 d}(\mathrm{Mo})$, depending on the proximity of $\mathrm{TeO}$, Fig. 3. As the $\mathrm{TeO}$ moiety was fixed at the grid points, we consider the energies shown in Fig. S3 as upper bounds of such configurations.

The configurations with the $\mathrm{TeO}$ moiety located close to $5 \mathbf{b}(\mathrm{Mo})$ or $8 \mathbf{d}(\mathrm{Mo})$ and the reducing electron relocated respectively, at relative energies of $\sim 35 \mathrm{~kJ} \mathrm{~mol}^{-1}$ with respect to the stable conformer $[\mathbf{2 , 2}][\mathbf{2}, \mathbf{2}]$, may be interpreted to approximate the $\mathrm{TeO}$ configurations $[\mathbf{2 , 5}]$ or $[\mathbf{2 , 8}]$. Because here we inspect only a single pentameric unit in contrast to the discussion above, we may compare these relative energies to values about half of those reported in Table 1. For example, configuration $\left[\mathbf{2 , 3},[\mathbf{3 , 2}]\right.$ is $40 \mathrm{~kJ} \mathrm{~mol}^{-1}$ above the Ref. [2,2][2,2], Table 1 , or about $20 \mathrm{~kJ} \mathrm{~mol}^{-1}$ per $\mathrm{TeO}$ moiety displaced towards sites $\mathbf{3}(\mathrm{V})$. These results suggest that the variations $[\mathbf{7 , 2}][\mathbf{7 , 2}],[\mathbf{2}, 7][\mathbf{7}, 2],[\mathbf{2}, 7][3,2]$, or $[\mathbf{2}, 3][3,2]$ are thermodynamically more accessible, with relative energies from $20 \mathrm{~mol}^{-1}$ to $33 \mathrm{~kJ} \mathrm{~mol}^{-1}$, than configurations involving the $\mathrm{TeO}$ configurations $[\mathbf{2 , 5}]$ or $[\mathbf{2 , 8}]$ just discussed.

The displacement of the $\mathrm{TeO}$ moiety causing a relocation of the polaron from $\mathbf{2 b}(\mathrm{Mo})$ to either $\mathbf{5 b}(\mathrm{Mo})$ or $\mathbf{8 d}(\mathrm{Mo})$ emphasizes that the proximity of $\mathrm{TeO}$ to a metal center may trigger a reduction of the latter.

\subsubsection{Atomic Charges and Spin Densities When the TeO Position Varies in the Hexagonal Channel}

We have just seen that the reducing electron at $\mathbf{2 b}(\mathrm{Mo})$ is relocated to other Mo sites of the $\mathrm{HC} \delta$ when the stabilizing effect of the $\mathrm{TeO}$ moiety is reduced at larger distances Te-Mo. To probe details of the associated charge transfer, we resorted to a slightly more approximate model, where the $\mathrm{TeO}$ moiety is displaced in a rigid fashion along a linear path in the (001) plane, from its stable position near $\mathbf{2 b}(\mathrm{Mo})$ in the configuration $[\mathbf{2}, \mathbf{2}][\mathbf{2 , 2}]$ to a position near center $\mathbf{3 b}(\mathrm{V})$ while keeping the location of all other centers fixed, Fig. 4. We chose this linear scan to limit the computational effort.

According to this model, the reducing electron is maintained at the site $\mathbf{2 b}(\mathrm{Mo})$ (spin density $\rho>0.7 e$ ) for $\mathrm{TeO}$ positions up to $90 \mathrm{pm}$ away from that starting location, Fig. 4b. Beyond that distance, the spin density drops to $\rho=0.25 e$, while the spin density at the center $8 \mathbf{d}(\mathrm{Mo})$ increases to $\rho=0.7 e$, indicating a concomitant shift of the reducing electron. Center $\mathbf{3 b}$ with $\mathrm{V}^{4+}$ is unable to host yet another reducing electron.

During that scan, the Hirshfeld charges q of the metal centers $\mathrm{Te}, \mathbf{2 b}(\mathrm{Mo})$, and $\mathbf{8 d}(\mathrm{Mo})$ exhibit trends concomitant to those of the spin densities $\rho$, Fig. $4 \mathrm{c}$. When the TeO moiety is moved $120 \mathrm{pm}$ from its initial position, the charge

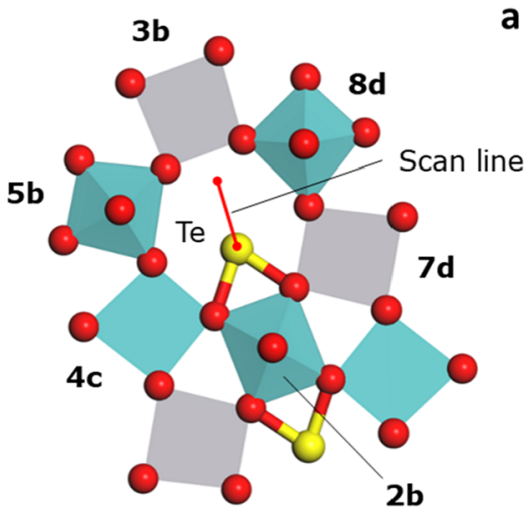

a
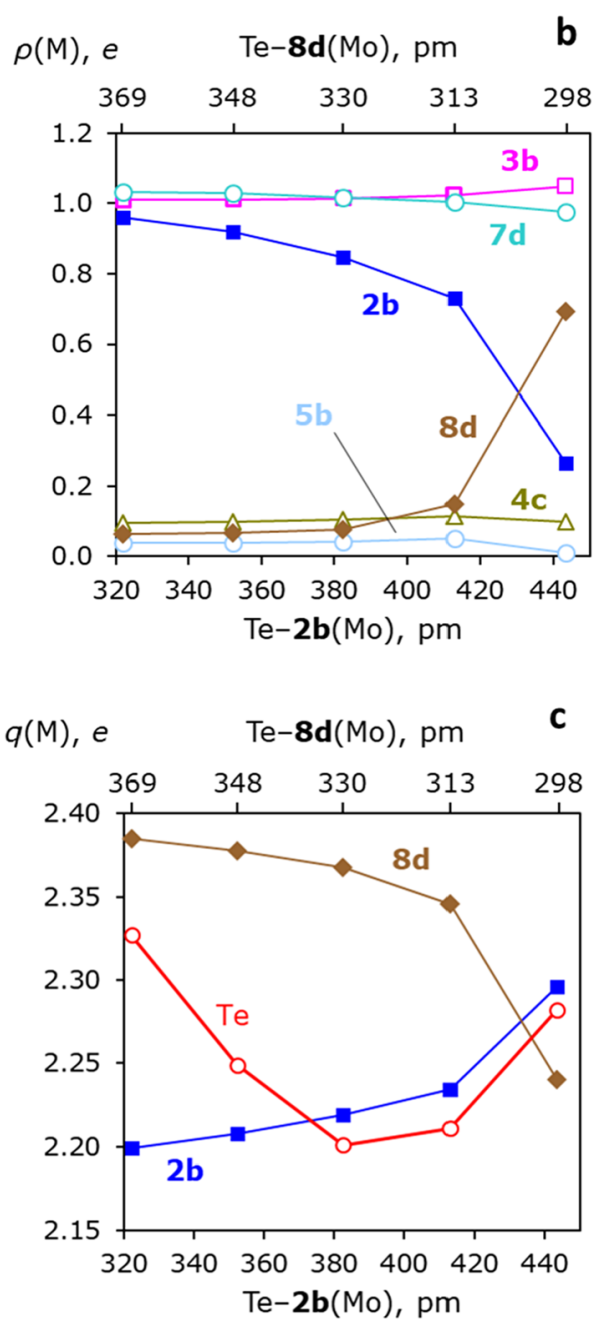

Fig. 4 a Scan line (red) for shifting the TeO moiety (as rigid structure) in the $\mathrm{HC} \delta$, connecting the initial configuration $[\mathbf{2 , 2}][\mathbf{2 , 2}]$ to a position close to the site $3 \mathbf{b}(\mathrm{V})$ where the equatorial Te-O bonds are $\sim 220 \mathrm{pm}$. In the initial configuration, $\mathrm{Mo}^{5+}$ at site $\mathbf{8 d}$ features a distorted, and $\mathrm{Mo}^{6+}$ at site $\mathbf{2 b}$ a highly symmetric local environment, see text. b Spin densities, $\rho$, of the metal sites $M$ forming the HC, when the $\mathrm{TeO}$ moiety is shifted along the scan line. c Similarly, Hirshfeld charges, $q(\mathbf{M}), \mathbf{M}=$ Mo at $\mathbf{2 b}$, Mo at $\mathbf{8 d}$, and Te, along the scan line 
of $2 \mathbf{b}(\mathrm{Mo})$ increases by $0.10 e$ while the charge of $\mathbf{8 d}(\mathrm{Mo})$ decreases by $0.14 e$, reflecting the transfer of the reducing electron. In the final position examined, the charge of $\mathbf{8 d}(\mathrm{Mo}), 2.24 e$, is lower than the charge of $\mathbf{2} \mathbf{b}(\mathrm{Mo}), 2.30$ $e$. Such a decreased charge at $\mathbf{8 d}(\mathrm{Mo})$ will lower the Te$\mathbf{8 d}(\mathrm{Mo})$ electrostatic interaction. Interestingly, the calculated charge of Te also changed quite remarkably. Starting from $2.33 e$ it reaches a minimum of $2.20 e$ at the distance $\mathrm{Te}-\mathbf{2 b}(\mathrm{Mo})=380 \mathrm{pm}$; thus, in that configuration the atomic charge of this Te species is quite similar to that of $\mathrm{Mo}^{5+}$ or $\mathrm{V}^{4+}$. This may be interpreted as a (partial) reduction of $\mathrm{Te}$ due to the missing equatorial oxygen centers, that are now outside the local environment of Te. It has been hypothesized $[17,31]$ that the loss of axial oxygen from the TeO moieties within the $\mathrm{HC}$, thus producing a reduced $\mathrm{Te}^{2+}$, causes a shortening of the Te-2 distance. By the same token, we expect a stronger interaction of partially reduced Te with its axial oxygen centers while increasing the equatorial $\mathrm{Te}-\mathrm{O}$ distances.

Next, we inspect the energies of the gap states along the scan line, Table S2 (Online Resource 1). The values $\varepsilon(\mathbf{2 b})$ and $\varepsilon(\mathbf{7 d})$ of the gap states are shifted to higher energies as Te is displaced towards $\mathbf{3 b}(\mathrm{V})$ across the HC. In contrast, $\varepsilon(3 \mathbf{b})$ shifts to lower energies when Te approaches $\mathbf{3 b}(\mathrm{V})$. Only when Te is at $443 \mathrm{pm}$ from $2 \mathbf{b}(\mathrm{Mo})$, or at $298 \mathrm{pm}$ from $\mathbf{8 d}(\mathrm{Mo})$, a peak with $d$ state character appears for $\mathbf{8 d}(\mathrm{Mo})$ at $-0.44 \mathrm{eV}$; its energy nearly matches that of the peak for the gap $d$ state at $\mathbf{2 b}(\mathrm{Mo}),-0.51 \mathrm{eV}$, Table S2 (Online Resource 1 ), as is to be expected for an electron transfer [60].

The symmetric and distorted local environments of the centers $2 \mathbf{b}(\mathrm{Mo})$ and $\mathbf{8 d}(\mathrm{Mo})$ were maintained during the model calculations just discussed. Thus, their equatorial Mo-O bonds remained at their initial values, in the ranges 198-202 $\mathrm{pm}\left(\mathrm{Mo}^{5+}\right)$ and $178-208 \mathrm{pm}\left(\mathrm{Mo}^{6+}\right)$, respectively. Thus, changes in the local metal environments are not required for changing the charge transfer between the sites $\mathbf{2 b}(\mathrm{Mo})$ and $\mathbf{8 d}(\mathrm{Mo})$ [or $\mathbf{5 b}(\mathrm{Mo})$ ]. This result clearly points to a Coulomb effect of the $\mathrm{TeO}$ moiety, to induce the electron transfer. Our rather simple model, naively assuming all other factors to remain constant, entails a change in energy by $\sim 100 \mathrm{~kJ} \mathrm{~mol}^{-1}$ as the charge $\mathrm{q}(2 \mathbf{b})$ changes from $2.30 e$ to $2.20 e$ at the $\mathbf{2 b}(\mathrm{Mo})$ center $320 \mathrm{pm}$ apart from Te with a charge of $2.33 \mathrm{e}$. Our model yields only a crude estimate, neglecting screening of charges as well as other interactions. Nevertheless, it provides a hint on the approximate size of this energy, despite the small change in the charge $\mathrm{q}(\mathbf{2 b})$.

The redistribution of the reducing electron as consequence of relocating the $\mathrm{TeO}$ moiety in the hexagonal channels is especially important, given the recent experimental evidence for the irregular positioning of Te columns within the hexagonal channels [59]. As described above, the reducibility of the metal centers, i.e., their ability to form a small polaron, increases with the proximity to a $\mathrm{TeO}$ moiety.
This may be rationalized as a stabilization due to a lowered $\mathrm{M}-[\mathrm{TeO}]$ electrostatic repulsion $(\mathrm{M}=\mathrm{V}, \mathrm{Mo})$, when the charge of $\mathrm{M}$ is somewhat reduced, when $\mathrm{M}$ carries a polaron.

\section{Conclusions}

We studied computationally how $\mathrm{TeO}$ moieties in the hexagonal channels (HCs) of the MoVNbTeO bulk material affect nearby transition metal sites, in particular in the pentameric unit. By populating sites $\mathbf{1}, \mathbf{3}$, and $\mathbf{7}$ with $\mathrm{V}$ ions and exploring various $\mathrm{TeO}$ placements within the $\mathrm{HCs}$, we examined how these $\mathrm{TeO}$ moieties affect the local structure of $\mathbf{2}(\mathrm{Mo})$ centers and induce alternative distributions of reduced metal centers.

- Various stable positions of $\mathrm{TeO}$ moieties within the hexagonal channels (HCs) near the sites $\mathbf{2}(\mathrm{Mo}), \mathbf{3}(\mathrm{V})$, and $7(\mathrm{~V})$ were determined to be energetically accessible by $20-33 \mathrm{~kJ} \mathrm{~mol}^{-1}$ uphill, translating to $40-66 \mathrm{~kJ} \mathrm{~mol}^{-1}$ uphill for both pentameric units per unit cell. These energy ranges characterize the manifold of stable positions of $\mathrm{TeO}$ within a channel, recently examined experimentally [59].

- Especially noteworthy is the unique result of our model calculations that site $2(\mathrm{Mo})$ at the center of the active site (pentameric unit) is reduced when symmetrically bracketed between adjacent $\mathrm{TeO}$ moieties. One each in the neighboring HCs. Any deviation from this special configuration $\mathrm{TeO}-2$ (Mo)- $\mathrm{TeO}$ induces the oxidation of Mo at site 2. This result adds a crucial detail about the condition to finding a reduced center, $\mathrm{Mo}^{5+}$, sometimes identified at site 2(Mo) [14, 16, 61].

Displacing a $[\mathrm{TeO}]^{2+}$ moiety in a $\mathrm{HC}$ may relocate the reducing electrons to another metal center in the wall of the $\mathrm{HC}$, to either $\mathbf{5}(\mathrm{Mo})$ or $\mathbf{8}(\mathrm{Mo})$. Yet, such alternative configurations are unfavorable by $\sim 35 \mathrm{~kJ} \mathrm{~mol}^{-1}$ per $\mathrm{TeO}$ displaced, in comparison to the symmetric arrangement where both $\mathrm{TeO}$ moieties are closely bracketing the 2(Mo) site of a pentameric unit.

- Previously we determined [28] that the likelihood of finding $\mathrm{V}^{5+}$ species at the sites in the wall of a $\mathrm{HC}$ increases if the channel holds a $\mathrm{TeO}$ defect. This is also important from a practical side, as decreasing the Te content leads to improved catalysts [11]. Our model study suggested that displacing the $\mathrm{TeO}$ moiety towards site $\mathbf{3}$, away from $\mathbf{2}(\mathrm{Mo})$, has a similar effect on $\mathbf{2}(\mathrm{Mo})$ than a $\mathrm{TeO}$ defect [28]. In both cases, 2(Mo) is oxidized and its local oxygen environment will be distorted, in agreement with the pseudo Jahn-Teller concept [57]. The propensity of Mo centers to be reduced, and thus to form "small polarons" [62], also increases near a $\mathrm{TeO}$ moiety. This points to a decisive role of $\mathrm{TeO}$ moieties at suitable positions, namely to control the ability to reduce site $2(\mathrm{Mo})$ at the 
center of the pentameric unit, discussed as active site $[4,21]$. Facilitating the mobility of $\mathrm{TeO}$ moieties will therefore directly impact the oxidation state of centers in the $\mathrm{HC}$. The combined occurrence of $\mathrm{V}^{4+}$ and $\mathrm{V}^{5+}$ within a pentameric unit has been discussed as crucial for an efficient oxidation catalyst $[8,21,26]$, hence highlighting the importance of tuning the distribution of reducing sites via locating $\mathrm{TeO}$ moieties at suitable distances or even the creation of $\mathrm{TeO}$ defects.

Supplementary Information The online version containssupplementary material available at https://doi.org/10.1007/s10562-021-03538-3.

Author Contributions JMA-R: Carried out calculations and the initial analysis, wrote the initial draft, carried out the illustrations. GR: Carried out calculations and contributed to the analysis. AG: Supervised the study and the analysis, revised the manuscript. NR: Designed the project, supervised the study and the analysis, and revised the manuscript.

Funding Open Access funding provided by TU Wien (TUW). This work was supported by Grant No. 1527700033 of the A*STAR Science and Engineering Research Council. The computational work profited enormously from a generous allotment of resources provided by the A*STAR Computational Resources Center and the National Supercomputing Centre Singapore.

Code Availability The computer codes applied are commercially available.

\section{Compliance with Ethical Standards}

Conflict of interest The authors declare that they have no conflict of interest.

Open Access This article is licensed under a Creative Commons Attribution 4.0 International License, which permits use, sharing, adaptation, distribution and reproduction in any medium or format, as long as you give appropriate credit to the original author(s) and the source, provide a link to the Creative Commons licence, and indicate if changes were made. The images or other third party material in this article are included in the article's Creative Commons licence, unless indicated otherwise in a credit line to the material. If material is not included in the article's Creative Commons licence and your intended use is not permitted by statutory regulation or exceeds the permitted use, you will need to obtain permission directly from the copyright holder. To view a copy of this licence, visit http://creativecommons.org/licenses/by/4.0/.

\section{References}

1. Ren T, Patel M, Blok K (2006) Energy 31:425-451

2. Amghizar I, Vandewalle LA, Van Geem KM, Marin GB (2017) Engineering 3:171-178

3. Hatano M, Kayo A (1991) Catalytic conversion of alkanes to nitriles, and a catalyst therefor, U.S. Patent 5049692

4. Grasselli RK, Burrington JD, Buttrey DJ, Desanto P, Lugmair CG, Volpe AF, Weingand T (2003) Top Catal 23:5-22

5. Grasselli RK (1999) Catal Today 49:141-153

6. Holmberg J, Grasselli RK, Andersson A (2004) Appl Catal A 270:121-134
7. Holmberg J, Hansen S, Grasselli RK, Andersson A (2006) Top Catal 38:17-29

8. Grasselli RK (2014) Catal Today 238:10-27

9. Celaya Sanfiz A, Hansen TW, Girgsdies F, Timpe O, Rödel E, Ressler T, Trunschke A, Schlögl R (2008) Top Catal 50:19-32

10. Sadakane M, Yamagata K, Kodato K, Endo K, Toriumi K, Ozawa Y, Ozeki T, Nagai T, Matsui Y, Sakaguchi N, Pyrz WD, Buttrey DJ, Blom DA, Vogt T, Ueda W (2009) Angew Chem Int Ed 48:3782-3786

11. Melzer D, Mestl G, Wanninger K, Zhu Y, Browning ND, Sanchez-Sanchez M, Lercher JA (2019) Nat Commun 10:4012

12. DeSanto P, Buttrey DJ, Grasselli RK, Lugmair CG, Volpe AF, Toby BH, Vogt T (2004) Z Kristallogr Cryst Mater 219:152-165

13. Wagner JB, Timpe O, Hamid FA, Trunschke A, Wild U, Su DS, Widi RK, Hamid SBA, Schlögl R (2006) Top Catal 38:51-58

14. Murayama H, Vitry D, Ueda W, Fuchs G, Anne M, Dubois JL (2007) Appl Catal A 318:137-142

15. Pyrz WD, Blom DA, Vogt T, Buttrey DJ (2008) Angew Chem Int Ed 47:2788-2791

16. Li X, Buttrey DJ, Blom DA, Vogt T (2011) Top Catal 54:614-626

17. Aouine M, Epicier T (2016) Millet J-MM. ACS Catal 6:4775-4781

18. Epicier T, Aouine M, Nguyen TT (2017) Millet J-MM. ChemCatChem 9:3526-3533

19. Lwin S, Diao W, Baroi C, Gaffney A, Fushimi R (2017) Catalysts 7:109

20. Trunschke A, Noack J, Trojanov S, Girgsdies F, Lunkenbein T, Pfeifer V, Hävecker M, Kube P, Sprung C, Rosowski F, Schlögl R (2017) ACS Catal 7:3061-3071

21. Grasselli RK, Lugmair CG, Volpe AF (2011) Top Catal 54:595-604

22. Naumann d'Alnoncourt R, Csepei L-I, Hävecker M, Girgsdies F, Schuster ME, Schlögl R, Trunschke A (2014) J Catal 311:369-385

23. Cheng M-J, Goddard WA (2016) Top Catal 59:1506-1517

24. Naraschewski FN, Jentys A, Lercher JA (2011) Top Catal 54:639-649

25. Fu G, Xu X, Sautet P (2012) Angew Chem Int Ed 51:12854-12858

26. Nguyen TT, Deniau B, Delichere P (2014) Millet J-MM. Top Catal 57:1152-1162

27. Grasselli RK, Volpe AF (2014) Top Catal 57:1124-1137

28. Arce-Ramos JM, Genest A, Rösch N (2020) J Phys Chem C 124:18628-18638

29. Hävecker M, Wrabetz S, Kröhnert J, Csepei L-I, Naumann d'Alnoncourt R et al (2012) J Catal 285:48-60

30. Chen X, Dang D, An H, Chu B, Cheng Y (2019) J Taiwan Inst Chem E 95:103-111

31. Zhu Y, Sushko PV, Melzer D, Jensen E, Kovarik L, Ophus C, Sanchez-Sanchez M, Lercher JA, Browning ND (2017) J Am Chem Soc 139:12342-12345

32. Li W, Fjermestad T, Genest A, Rösch N (2018) Cat Sci Technol 8:2654-2660

33. Lide DR, in: Handbook of Chemistry and Physics, eds. Lide DR (CRC Press LLC, Boca Raton, Florida, 2003), ch. 10

34. Dovesi R, Orlando R, Erba A, Zicovich-Wilson CM, Civalleri B, Casassa S, Maschio L, Ferrabone M, De La Pierre M, D’Arco P, Noël Y, Causà M, Rérat M, Kirtman B (2014) Int J Quantum Chem 114:1287-1317

35. Becke AD (1993) J Chem Phys 98:5648-5652

36. Grimme S (2006) J Comput Chem 27:1787-1799

37. Dinda S (2017) Chiu C-c, Genest A, Rösch N. Comput Theor Chem 1101:36-45

38. Rugg G, Genest A, Rösch N (2018) J Phys Chem A 122:7042-7050

39. Hu Z, Metiu H (2011) J Phys Chem C 115:5841-5845 
40. Chiu C-C, Vogt T, Zhao L, Genest A, Rösch N (2015) Dalton Trans 44:13778-13795

41. Monkhorst HJ, Pack JD (1976) Phys Rev B 13:5188-5192

42. Hirshfeld FL (1977) Theor Chim Acta 44:129-138

43. Li W-Q, Fjermestad T, Genest A, Rösch N (2019) Cat Sci Technol 9:1559-1569

44. Ruiz E, Llunell M, Alemany P (2003) J Solid State Chem 176:400-411

45. Bredow T, Jug K, Evarestov RA (2006) Phys Status Solidi B 243:R10-R12

46. Hay PJ, Wadt WR (1985) J Chem Phys 82:270-283

47. DallOlio S, Dovesi R, Resta R (1997) Phys Rev B 56:10105-10114

48. Heyd J, Peralta JE, Scuseria GE, Martin RL (2005) J Chem Phys 123:174101

49. Kresse G, Hafner J (1993) Phys Rev B 47:558-561

50. Kresse G, Hafner J (1994) Phys Rev B 49:14251-14269

51. Kresse G, Furthmüller J (1996) Comput Mater Sci 6:15-50

52. Kresse G, Furthmüller J (1996) Phys Rev B 54:11169-11186

53. Perdew JP, Burke K, Ernzerhof M (1996) Phys Rev Lett 77:3865-3868

54. Dudarev SL, Botton GA, Savrasov SY, Humphreys CJ, Sutton AP (1998) Phys Rev B 57:1505-1509

55. Buttrey DJ, Blom DA, Vogt T, in: Complex Oxides, An Introduction, eds. Vogt T, Buttrey DJ (World Scientific, Singapore, 2019), ch. $6: 157-198$
56. Lunkenbein T, Girgsdies F, Wernbacher A, Noack J, Auffermann G, Yasuhara A, Klein-Hoffmann A, Ueda W, Eichelbaum M, Trunschke A, Schlögl R, Willinger MG (2015) Angew Chem Int Ed 54:6828-6831

57. Bersuker IB (2013) Chem Rev 113:1351-1390

58. Kunz M, Brown ID (1995) J Solid State Chem 115:395-406

59. Lunkenbein T, Masliuk L, Plodinec M, Algara-Siller G, Jung S, Jastak M, Kube P, Trunschke A, Schlögl R (2020) Nanoscale 12:6759-6766

60. Atkins PW, De Paula J, in: Atkins' Physical Chemistry (10th ed.), (Oxford University Press, Oxford, New York, 2014), ch. 21

61. Govindasamy A, Muthukumar K, Yu J, Xu Y, Guliants VV (2010) J Phys Chem C 114:4544-4549

62. Shluger AL, Stoneham AM (1993) J Phys 5:3049-3086

63. Melzer D, Xu P, Hartmann D, Zhu Y, Browning ND, SanchezSanchez M, Lercher JA (2016) Angew Chem Int Ed 55:8873-8877

Publisher's Note Springer Nature remains neutral with regard to jurisdictional claims in published maps and institutional affiliations. 